\section{Intensity Fluctuations of Acoustic Noise After Strong Nonlinear Amplification}

\author{
H. Kabelka and H. Kuzmany
}

Ludwig Boltzmann Institut für Festkörperphysik und II. Phys. Institut der Universität Wien

(Z. Naturforsch. 32 a, 1056-1058 [1977] ; received April 19, 1977)

The intensity fluctuations of nonlinearly amplified phonons have been investigated by high resolution photon counting in a Brillouin scattering experiment. No significant signal to noise transition could be observed.

Nonlinear interaction of waves can lead to a drastic change of their statistical properties. This was demonstrated extensively for optical waves in connection with laser theories and corresponding experiments using photon counting techniques ${ }^{1-4}$. For this case it was shown that the random Gaussian distribution of the fluctuations of the intensity of thermal light is transformed into a coherent wave where the probability distribution of the intensity is a $\delta$-function. The resulting photon counting distribution are the Bose distribution and the Poisson distribution, respectively ${ }^{5}$.

In the case of acoustic phonons it was suggested by several authors ${ }^{6-10}$ to use the acoustoelectric effect in piezoelectric semiconductors for a strong nonlinear amplification of the phonons. In order to obtain a correct counting distribution from such experiments, the experimental set up must satisfy the condition:

$$
v_{\mathrm{s}} \alpha \tau \ll 1, \quad \Delta \omega \tau \leqq 1, \quad \Delta v \tau \ll 1, \quad \tau \leqq \tau_{0}
$$

where $v_{\mathrm{s}}, \alpha, \tau, \Delta \omega, \Delta v$, and $\tau_{0}$ are the sound velocity, the amplification constant, the time interval for the counting, the band width of the phonons from which the light is scattered, the band width of the laser and the life time of the phonons, respectively. It was pointed out explicitly by Gantsevich et al. ${ }^{10}$ that under these conditions a transformation of incoherent phonon flux into a coherent acoustic signal should occur for nonlinear amplification. On the other hand, Wonneberger and Lempert ${ }^{9}$ discussed the problem with inclusion of the mutual interaction of the phonons and found that the distribution of the intensity fluctuations should follow a $\beta$ distribution.

The only experimental results to this problem have so far been reported by Siebert and Wonneberger ${ }^{8,11}$. Unfortunately, in this experiment not all of the conditions (1) were satisfied. Especially the

Reprint request to Doz. Dr. H. Kuzmany, II. Phys. Inst. d. Univ. Wien, Strudlhofgasse 4, A-1090 Wien, Austria. second condition in (1) was not satisfied by a factor of 300 due to the large band width $\Delta \omega=$ $1 \times 10^{9} \mathrm{sec}^{-1}$ and the long counting interval of $300 \mathrm{nsec}$. Also relatively large statistical counting errors were included. It was therefore desirable to obtain more exact results with an experimental set up satisfying the full set of condition (1).

We report investigations on $\mathrm{ZnO}$ and $\mathrm{CdS}$ obtained with a krypton laser as a light source and an on-line automatically reading photon counting system. High acoustic flux was excited in the semiconducting crystals by application of drift field pulses of $1-33 \mu \mathrm{sec}$ duration. The Brillouin scattering signal was detected under the condition of momentum conservation and with an aperture of only $1.5 \times 10^{-4} \mathrm{rad}$ corresponding to a band width $\Delta \omega=1 \times 10^{7} \mathrm{sec}^{-1}$. The detecting system consisted of a photomultiplier connected to an amplifier discriminator and an SSR photon counter gated externally with gate times from 150 to 500 nsec. The dead time $T$ of the system was determined experimentally to be 20 nsec. The output of the photon counter was fed into a computer of type HP 9820, which was programmed as a multichannel analyser. Each distribution of the intensity fluctuations was determined from at least $4 \times 10^{4}$ tests which is better by a factor of 20 as compared to the results in ${ }^{8}$. A detailed description of the experimental set up will be given elsewhere ${ }^{12}$. The first three conditions of (1) were met by working at a high excitation level where $\alpha$ is small, at a suitable narrow aperture and using a single mode laser. The last condition will be discussed below.

Single crystals of $\mathrm{ZnO}$ from the Airtron Corporation and of CdS from the Eagle Picher Company have been used. The probability $p(N)$ of counting $N$ photons within the time interval $\tau$ has been determined. Results for $\mathrm{ZnO}$ and $\mathrm{CdS}$ were similar, so that only the results for the $\mathrm{ZnO}$ crystals will be discussed below. The crystals were driven in a mode where the acoustic flux is piled up at the anode and the experimental parameters are:

$$
f_{\mathrm{s}}=5.5 \mathrm{GHz}, \quad l=6.4 \mathrm{~mm}, \quad b=1.7 \mathrm{~mm},
$$

$d=1.4 \mathrm{~mm}, \quad E=1.9 \mathrm{kV} / \mathrm{cm}, \quad n=2.5 \times 10^{15} \mathrm{~cm}^{-3}$,

$$
\mu=180 \mathrm{~cm}^{2} / \text { Vsec. }
$$

The experimental results will be discussed using the reduced factorial moments $H_{k}$ of the distributions defined as

$$
H_{k}=\left[\sum_{N=0}^{\infty} N(N-1) \ldots(N-k+1) p(N)\right] /\langle N\rangle^{k}
$$

where $k$ is the order of the moment. Unfortunately, a dead time correction for the experimental results 
cannot be performed since the influence of the counting intervals with high counting rates is too large. Dead time correction can only be performed to the various theoretical counting distributions and the corrected distribution can be compared with the experiments. We have used the theory of Arecchi and Degiorgio ${ }^{1}$ for the dead time correction according to which the corrected distribution $p$ is obtained from the uncorrected $p^{\prime}$ by the relation

$$
p(N)=\sum_{M=N}^{n} P(N, M) \cdot p^{\prime}(M)
$$

where

$P(N, M)=\left(\begin{array}{c}M-1 \\ M-N\end{array}\right) \sum_{i=M-N}^{M}(-1)^{i}\left(\begin{array}{c}M \\ i\end{array}\right) a^{M-i} \varepsilon^{i} \sigma_{i, M-N}$ $\sigma_{i r}=\sum_{j=1}^{r}(-1)^{j}\left(\begin{array}{l}r \\ j\end{array}\right) j^{i}, \quad a=1-\varepsilon(N-1), \quad \varepsilon=T / \tau$

and $n$ is the highest possible photoelectron counting number for the given value of $\varepsilon$.

Figure 1 shows the values of the factorial moments of Bose distributions (curve 1) and of multiple convolved Bose distributions (curves 2 to 5 ) as a function of their order. Curve 5 is already nearly identical to a Poisson distribution. The fully drawn lines are experimental results for $\mathrm{ZnO}$ corresponding to counting intervals indicated in nsec as a parameter. The statistic of the intensity fluctuation is obviously somewhere intermediate between the statistic of thermal noise and that of a coherent signal. However, its position might still be a function of the counting interval $\tau$ since by convolution of the Bose distribution a transition from the Bose to the Poisson distribution is established ${ }^{7}$, (curves 2 to 5 in Figure 1).

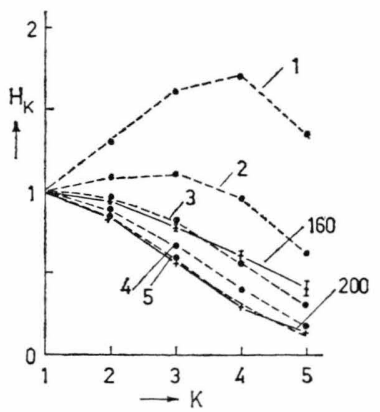

Fig. 1. Calculated values of the $k^{\text {th }}$ reduced factorial moments $H_{k}$ of the Bose distribution (dashed curve 1) and convolved Bose distributions. Curves 2, 3, 4, and 5 correspond to 2-fold, 4-fold, 8-fold, and 16-fold convolution, respectively. All calculated factorial moments are dead time corrected leading to the characteristic decrease with increasing order. The fully drawn lines are the experimental results for $\mathrm{ZnO}$. The labels 160 and 200 are the counting time intervals in nsec.
The best fit to the experimental result for $\tau=$ 160 nsec was obtained with a four fold convolution of a Bose distribution. From this point of view an increase of the counting interval $\tau$ should result in a shift of the distribution towards the Poisson distribution which was observed indeed as can be seen in Fig. 1 for $\tau=200$ nsec. A reduction of the counting interval below $150 \mathrm{nsec}$ was not possible because of the dead time of the counting system. However, since the experimental results are well represented by the convolved Bose distribution the coherence time should be of the order of 40 nsec. This is of the same order of magnitude as the attenuation constant for the corresponding thermal phonons ${ }^{13}$. Thus discussing te experimental results in the approximation of a Bose distribution, no noise to signal transition was observed in contrast to the prediction in Ref. ${ }^{8}$ and ${ }^{10}$.

With respect to the theory of Wonneberger the photon counting distribution is given by the relation:

$$
\begin{gathered}
p(N)=\left(\frac{N_{m}}{N_{m}^{\prime \prime}}\right)^{N} \frac{\langle N\rangle^{N}}{N !} \frac{\left(N_{m}-1\right) !\left(N_{m}^{\prime \prime}+N-1\right) !}{\left(N_{m}^{\prime \prime}-1\right) !\left(N_{m}+N-1\right) !} \\
\cdot{ }_{1} F_{1}\left(N_{m}^{\prime \prime}+N, N_{m}+N, \frac{N_{m}}{N_{m}^{\prime \prime}}\langle N\rangle\right)
\end{gathered}
$$

where $N_{m}$ and $N_{m}^{\prime \prime}$ are the number of interacting and scattering modes, respectively, and ${ }_{1} F_{1}$ is the confluent hypergeometric function. The factorial moments of this distribution have been calculated numerically for various parameters $N_{m}$ and $N_{m}^{\prime \prime}$, respectively. In all cases a dead time correction according to Eq. (3) was performed. Good agreement between theory and experiment was obtained but the fitting was possible with more than one set of parameters $N_{m}$ and $N_{m}^{\prime \prime}$. In Fig. 2 the experimental results for $\mathrm{ZnO}$ are compared with a calculation



Fig. 2. Calculated values of the $k^{\text {th }}$ reduced factorial moments $H_{k}$ as calculated from Eq. (4) for parameters $N_{m}$ and $N_{m}^{\prime \prime}$ as indicated. Dead time corrections are included as obtained from Equation (3). The fully drawn line is the same experimental result as in Fig. 1, corresponding to a counting interval of $160 \mathrm{nsec}$. 
using the values 16 and 3 for $N_{m}$ and $N_{m}^{\prime \prime}$, respectively.

Though this result is not typical for a Bose distribution it should be kept in mind, that the ratio of the coherence length to the counting interval might also influence the distribution function in this case. Fitting the experimental result for $\tau=$ $200 \mathrm{nsec}$ to the theoretical curves gives $N_{m}^{\prime \prime}=8$ which is only slightly larger than the value expected from multiple convolution of Bose distributions. The reason might be, that the $\beta$-distribution is only equivalent to the convolved Bose distributions if $N_{m}$ is very large.

In conclusion, we have observed the intensity fluctuations of nonlinear amplified acoustic noise. The use of a very small pinhole and a large distance between the photomultiplier and the scattering crystal on the one hand, and an automatically reading photon counting system on the other hand, provided accurate and reliable results for a comparison with theoretical models. In the simplified model of convolved Bose distributions a coherence length of $40 \mathrm{nsec}$ was found for $\mathrm{ZnO}$. Using the $\beta$-distribution for a description of the intensity fluctuations revealed a couple of pairs of parameters which describe the experimental results. The results do not necessarily indicate a noise to signal transition.

The authors acknowledge Prof. K. Seeger for his continual interest in the progress of this work and Prof. Wonneberger for many valuable discussions.

This work was supported by the Ludwig Boltzmann Gesellschaft zur Förderung der wissenschaftlichen Forschung in Österreich and by the Fonds zur Förderung der wissenschaftlichen Forschung.
${ }^{1}$ F. T. Arecchi, Proc. of the Int. School of Physics "Enrico Fermi", p. 57, Varena Academic Press, New York and London 1969.

2 H. Haken, Laser Handbook, p. 115, Amsterdam, North Holland, Eds. Arecchi and E. O. Schulz-Dubois, 1972.

3 F. T. Arecchi and V. Degiorgio, Phys. Rev. A 3, 1108 [1971].

4 C. L. Mehta, Progress in Optics 8, 373 [1969], Ed. E. S. Wolf.

5 F. T. Arechi, Phys. Rev. Lett. 15, 912 [1965].

${ }^{6}$ H. Kuzmany, phys. stat. sol. (a) 25, 9 [1974].
7 F. Siebert, Dissertation, Univ. Freiburg/BRD 1970.

8 F. Siebert and W. Wonneberger, Phys. Lett. 37 A, 367 [1971].

9 W. Wonneberger and J. Lempert, Z. Naturforsch. 28 a, 762 [1973].

10 S. V. Gantsevich, V. L. Gurevich, V. D. Kagan, and R. Katilius, phys. stat. sol. (b) 75, 407 [1976].

11 W. Wonneberger, J. Lempert, and W. Wettling, J. Phys. C: Solid State Phys. 7, 1428 [1974].

12 H. Kabelka and H. Kuzmany, to be published.

13 M. F. Lewis, J. Acoust. Soc. Amer. 43, 852 [1968]. 